\title{
De-coupling Anelastic and Elastic Deformation in Metallic Glass Thin Films via Measurement of Micro Strain Tensors Using in situ Electron Diffraction.
}

\author{
Rohit Sarkar ${ }^{1}$, Christian Ebner ${ }^{2}$, Christian Rentenberger ${ }^{2}$, Jagannathan Rajagopalan ${ }^{1}$. \\ 1. School of Engineering for Matter, Transport and Energy, Arizona State University, Tempe. USA. \\ 2. Physics of Nanostructured Materials, Faculty of Physics, University of Vienna, Vienna, Austria.
}

Several techniques have been recently developed to measure the lattice strain of crystalline materials using the TEM [1]. The methods employ either (i) High resolution TEM [2] and dark filed electron holography [3] or (ii) nano beam electron diffraction [4] and convergent beam electron diffraction [5]. However, though metallic glasses have been studied extensively using the TEM, measurements of micro-strains in these materials have not been carried out. Micro strains in metallic glasses has only been evaluated by techniques that employ high energy x-ray and neutron diffraction[6]. These techniques involve calculating the micro strain tensors by measuring the relative shift of diffraction peaks in reciprocal space during straining. Such techniques are limited to bulk specimen and lack the ability to monitor changes in the microstructure using high resolution imaging. In our work we use a novel in situ TEM technique described in [7] to measure local micro strain tensors in amorphous TiAl thin films.

The technique measures geometric changes in the first amorphous ring of Selected Area Diffraction (SAD) pattern during in situ straining to compute the micro strain tensors. The freestanding thin films are strained in situ using a unique MEMS based tensile testing stage [8] and SAD patterns are acquired from regions of $1.2 \mu \mathrm{m}$ diameter. The applied strain causes ellipticity to appear in the SAD pattern which is measured using a custom built script to compute the micro strain. The macro stress and strain are calculated by measuring the deflection of built-in gauges of the MEMS device. While the stressstrain response was found to be linear, the micro strain $\left(\mathrm{e}_{1}\right)$ was found to be consistently smaller in magnitude than the macro strain $\left(\varepsilon_{\text {macro }}\right)$. This difference in magnitude indicated the presence of anelastic deformation in the metallic glass films. Since the SAD pattern geometry is sensitive only to the elastic strain tensors, the $\mathrm{e}_{1}$ value would not take into account any anelastic deformation taking place in metallic glass specimen. The existence of anelastic deformation was further verified by measuring the strain rate dependence of the stress-strain response of the film. Additionally, the applied strain was found to narrow the spread of the nearest neighbor atomic distances as indicated by a permanent reduction in the FWHM of the SAD amorphous ring.

We show that in situ electron diffraction can be used to detect deformation induced structural rearrangements and reveal the presence of anelastic deformation in metallic glass thin films. This technique gives us the unique capability to measure micro strain tensors in precise locations of nanostructured specimen with sub-micrometer resolution while being able to monitor deformation induced microstructural changes. This would enable a more extensive analysis of the deformation processes in metallic glasses [9].

References:

[1] A. Béché, J.L. Rouvière, J.P. Barnes, D. Cooper, Ultramicroscopy. 131 (2013) 10-23.

[2] F. Hüe, M. Hÿtch, H. Bender, F. Houdellier, A. Claverie, Phys. Rev. Lett. 100 (2008) 156602. 
[3] A. Béché, J.L. Rouvière, J.P. Barnes, D. Cooper, Ultramicroscopy. 111 (2011) 227-238.

[4] K. Usuda, T. Numata, T. Irisawa, N. Hirashita, S. Takagi, Materials Science and Engineering: B. 124-125 (2005) 143-147.

[5] L. Clément, R. Pantel, L.F.T. Kwakman, J.L. Rouvière, Applied Physics Letters. 85 (2004) 651-653.

[6] H.F. Poulsen, J.A. Wert, J. Neuefeind, V. Honkimäki, M. Daymond, Nat Mater. 4 (2005) 33-36.

[7] C. Ebner, R. Sarkar, J. Rajagopalan, C. Rentenberger, Ultramicroscopy. In Review (2016).

[8] J.H. Han, M.T.A. Saif, Review of Scientific Instruments. 77 (2006) 045102.

[9] This project was funded by the National Science Foundation (NSF) grants ECCS 1102201, CMMI 1400505 and DMR 1454109. The authors would like to gratefully acknowledge the use of facilities at the John M. Cowley Centre for High Resolution Electron Microscopy and the Centre for Solid State Electronics Research at Arizona State University and at the Faculty of Physics of the University of Vienna. Financial support from the Austrian Science Fund (FWF): [P22440, I1309] is acknowledged.
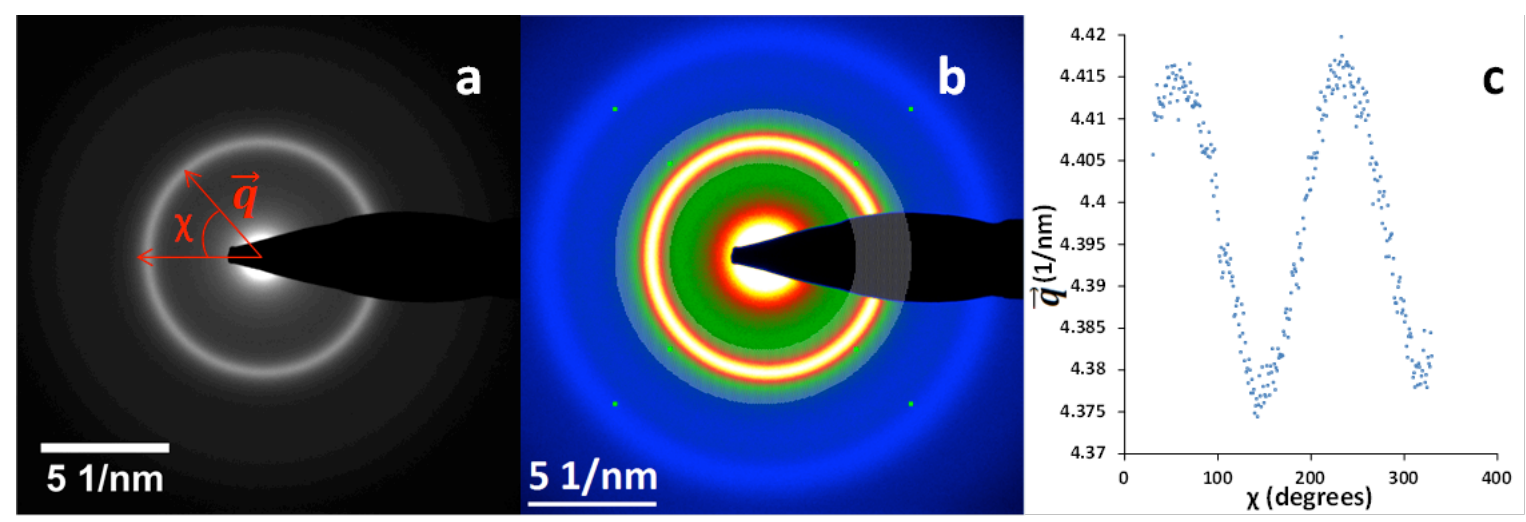

Figure 1. a) SAD pattern of the TiAl film showing the amorphous ring and the corresponding reciprocal lattice vector $q$ at an angle $\chi$. (b) The ellipse being fitted to the SAD pattern to accurately determine the center using the Digital Micrograph ${ }^{\mathrm{TM}}$ software. (c) A plot showing the variance of $\mathrm{q}$ at different angles $\chi$ along the SAD ring upon straining. We observe that the q value increases perpendicular to the loading direction and decreases parallel to it causing ellipticity in the SAD pattern. 